O.S. Logacheva, O.V. Kozhevnikova, A.E. Pal'tseva, L.S. NamazovaBaranova, A.K. Gevorkyan

Scientific Center of Children's Health, Moscow, Russian Federation

\title{
Modern methods of indentifying the predictors of cardio-vascular diseases' in children at early stages
}

\section{Author affiliation:}

Logacheva Olga Sergeevna, MD, doctor at the instrumental and laboratory diagnostics department of research institute of preventive pediatrics and medical rehabilitation's consultative-diagnostic center of at SCCH, RAMS

Address: 2/1, Lomonosovskii ave., Moscow, 119991, tel.: +7 (495) 967-14-20

Article received: 04.11.2012, accepted for publication: 24.03.2013.

Cardiovascular diseases are the main cause of invalidism and untimely death of people in developed countries. The share of cardiovascular diseases in the mortality rate reaches $40-60 \%$. The frequency rate of cardiovascular diseases among children and adolescents is growing with each passing year. Increased blood pressure variability in patients with arterial hypertension in youth is a factor aggravating the arterial stiffness derangement.

The aim of this study is to develop methods of early cardiovascular diseases' diagnostics in children in order to reduce the rate of primary and repeated complications in the future. Observation showed that singular blood pressure measurements at a consultative appointment with a doctor do not give the full picture of the character of its changes in a child and may lead to incorrect diagnosis, as blood pressure has considerable individual variability. Daily blood pressure monitoring allowed revealing high arterial hypertension rate in children at night, including children with normal and low blood pressure during daytime. It is shown that the non-invasive oscillometric arteriography method allows to 
determine parameters characterizing arterial vessels' remodeling with a high reproducibility. Significant difference between the obesity degree and the pulse wave aortic spread speed increase has been indentified.

Keywords: cardiovascular diseases, atherosclerosis, children, blood pressure, daily blood pressure monitoring, non-invasive arteriography, pulse wave's aortic spread speed, augmentation index.

Mortality from cardiovascular diseases in Russia makes 56\% out of total mortality rate. Along with such well-known factors of cardiovascular disease development and mortality risk as increased blood pressure (BP), diastolic dysfunction, left ventricle hypertrophy, altered lipid pattern, compromised heredity, obesity, arterial stiffness alteration has been gaining importance recently.

In modern cardiology there is a concept of a single cardiovascular continuum - continuous development of cardiovascular diseases: from risk factors to chronic cardiovascular collapse development. That is why researchers are now interested in finding ways to reveal functional cardiac and vascular changes in childhood and adolescence on the stage of transition or borderline states, when the disease does not yet manifest itself in its classical form [1, 2].

There are literary data demonstrating close relationship of the frequency of cardiovascular complications and condition of great vessels [3]. The most attractive techniques for assessing arterial condition are the non-invasive, available and easily reproducible ones.

One of the prevention areas is the detection of preclinical stages of atherosclerotic process, which starts forming in childhood. At the same time, early atherosclerosis development processes in children and adolescents have not been sufficiently studied yet [4].

It is known that singular BP measurements do not reveal its real level [5-7]. Ambulatory blood pressure monitoring (ABPM) is now widely used when diagnosing hypertension/hypotension and assessing therapy efficacy. 
ABPM was conducted in 477 children (307 boys and 170 girls) of 9-17 years of age. Patients were referred to ABPM by different specialists: cardiologist, endocrinologist, neurologist and pediatrician - concerning the revealed BP alterations with both upward and downward bias. This group did not involve children with endocrine system pathology. ABPM was conducted using devices “TM-2421” (A\&D, Japan) and "DR-102” (Schiller, Switzerland). BP was measured 30 minutes apart in the day and at night by 2 methods: oscillometric and auscultative. While being monitored, children remained at home observing the day off regimen and kept a diary of physical activity and complaints (antihypertensive measures were not used in any of the cases).

Singular measurements at a consultative visit to a pediatrician do not give the full presentation of the blood pressure alteration character in a child and may lead to an incorrect diagnosis, since BP has considerable individual variability. It is difficult to diagnose arterial hypertension/hypotension on early stages of their development, when they usually take an asymptomatic course. Ambulatory BP monitoring technique is characterized by high reproducibility, minimizes the "white coat" phenomenon's influence on the BP level, which is often observed in adolescents characterized by nervous system's lability. Blood pressure fluctuations associated with emotions often lead to arterial hypertension hyperdiagnostics and unreasonable therapy.

It has been established using chronobiological analysis that blood pressure levels in children have considerable individual variability in different days of week, which is why singular BP measurements at a consultative visit to a pediatrician do not allow adequately assessing tendency and dynamics of BP alterations in children; this leads to ambiguity in determining vegetative dysfunction types. Mean values of diurnal BP rhythms' parameters in adolescents of 13-15 years of age differ by days of week and more pronounced in overweight children. It has been revealed that increase in the body weight index percentile in children and adolescents correlates with the increase in mean levels of systolic and diastolic pressure, hypertension time and square. Increase in the body weight index 
percentile indicates the increase in the risk of arterial hypertension formation in children. It has been proved that night diastolic hypertension in school age children is connected with obesity. Increased variability of BP levels at night in children with night hypertension and normal BP indices in the day is only revealed by ambulatory blood pressure monitoring and allows accurately diagnosing mixed vegetative dysfunction syndrome.

The necessary components of cardiovascular continuum are endothelial system's imbalance and cardiovascular remodeling processes. That is why the role of structure functional arterial alterations in the development of blood circulation system diseases increases. Vascular wall's morphological structure failure indicates arterial remodeling - a prognostic factor of cardiovascular complications' development and an independent predictor of future "catastrophes” [3].

Pulse wave velocity is of the highest significance for assessing arterial remodeling using non-invasive diagnostics methods. The values of pulse wave velocity and augmentation index were for the first time determined in the population of Russian children while conducting non-invasive oscillometric arteriography.

According to the study goals and objectives, data of 514 patients of 3-13 years of age were analyzed; 363 out of them were apparently healthy (184 girls and 179 boys) and 151 were overweight or had the I-III degree of constitutiveexogenous obesity. Non-invasive oscillometric arteriography was conducting using the device "TensioMed” (Hungary). The prerequisites for measurement accuracy were rest before and during the study. BP values should have corresponded to normal values.

Mean reference non-invasive arteriography values - augmentation index and aortic pulse wave velocity - were obtained (tb. 1, 2).

The authors showed that the non-invasive arteriography method allows determining values characterizing arterial vascular remodeling with high reproducibility. 
It has been revealed that pulse wave velocity and augmentation index do not depend on a child's sex. Non-invasive arteriography indices are in direct correlation to age and body length. The data obtained in children are significantly different from the standardized norms of pulse wave velocity in adults, which is why it is necessary to go by age-adequate values.

Significant dependence between obesity degree and vascular wall remodeling intensification has been determined. Pulse wave velocity depends on the obesity degree, and the bigger the body weight the higher the velocity (pic.).

The most significant increase in pulse wave velocity (PWV) is in patients with II-III degree of obesity. Direct correlation between PWV indices' increase and factors aggravating arterial remodeling (blood serum's total cholesterol level and "fatty hepatosis" hepatic affection) has been established in children with different obesity degree.

The obtained results indicate that augmentation index and aortic pulse wave velocity values were higher in children with the increased body weight and hypercholesterolemia than in the group without hematologic changes; this proves the fact of vascular remodeling at hypercholesterolemia. It has been noted that body weight parameters, body weight index, aortic pulse wave velocity and augmentation index were higher in children with fatty hepatosis than in healthy children.

The obtained results correlate with literary data on the European children's population. This allows concluding that it is possible to use non-invasive arteriography method in the Russian children's population in order to reveal early signs of the developing atherosclerotic vascular alterations.

The obtained data were used to formulate early (preclinical) criteria of diagnosing atherosclerotic vascular alterations' manifestations in children and were introduced into ambulatory practice.

Using the non-invasive arteriography method in order to determine pulse wave velocity and augmentation index allows evaluating as simply and accurately as possible the arterial wall's condition, notably, its structural-morphological 
alterations leading to elasticity reduction and increase in stiffness and vascular resistance.

Oscillometric arteriography method is non-invasive, is notable for simplicity and is maximally automatized. Analysis does not take more than 10-12 minutes, which is why this method is efficient for screening tests and may be used in ambulatory pediatric practice. Reliability and credibility of oscillometric arteriography allow revealing high risk groups of cardiovascular pathology.

Primary prevention of cardiovascular diseases conducted in the population in tote proved its efficacy. Non-invasive oscillometric arteriography method will make it more effective, as its use will allow distinguishing a group of high risk patients from the general population.

The developed algorithm of diagnosing early markers of cardiovascular diseases developing in children in ambulatory conditions predetermined new approaches to their therapy, prevention and correction.

\section{REFERENCES}

1. Oganov R.G. Kardiologiya - Cardiology. 1991; 31(5): 5-7.

2. Belenkov Yu.N., Mareev V.Yu. Serdechnaya nedostatochnost' - Heart Failure. 2002; 3 (1): 7.

3. Weber K.T., Anversa P., Armstrong P.W., Brilla C.G., Burnett J.C.Jr., Cruickshank J.M., Devereux R.B., Giles T.D., Korsgaard N., Leier C.V. et al. Remodeling and reparation of the cardiovascular system. J Am Coll Cardiol. 1992; 20: 3-16.

4. Aleksandrov A.A. Kardiologiya - Cardiology. 1993; 8: 67-72.

5. Soergel M., Kirschstein M., Busch C., Danne T., Gellermann J., Holl R., Krull F. et al. Oscillometrc ywenty-four-hour ambulatory blood pressure values in healthy children and adolescent: A multicenter trial including 1141 subjects. J Pediatrics. 1997; 130: 178-184.

6. Petrov V.I., Ledyaev M.Ya. Arterial'naya gipertenziya u detei i podrostkov [Hypertension in Children and Adolescents]. Volgograd, 1999. 130 p. 
7. Logacheva O.S., Kozhevnikova O.V., Namazova-Baranova L.S., Pal'tseva A.E., Ryzhkova L.A., Shirokova I.V. Pediatricheskaya farmakologiya - Pediatric pharmacology. 2009; 6 (5): 38-41.

Table 1. Reference augmentation index values (AIX, \%) in correlation to age

\begin{tabular}{|l|c|c|c|c|c|c|c|c|}
\hline \multicolumn{7}{|c|}{ AIX, \% } \\
\hline Age (years) & $\mathrm{N}$ & Min & Max & \multicolumn{5}{|c|}{$\%$} \\
\cline { 5 - 9 } & & & & 10 & $\mathbf{2 5}$ & $\mathbf{5 0}$ & $\mathbf{7 5}$ & 90 \\
\hline $3-5$ & 35 & -69.23 & -27.45 & -63.95 & $\mathbf{- 5 0 . 3 7}$ & $\mathbf{- 4 5 . 3 1}$ & $\mathbf{- 3 8 . 6 1}$ & -33.04 \\
\hline 6 & 35 & -67.78 & -31.17 & -58.22 & $\mathbf{- 5 3 . 6 8}$ & $\mathbf{- 4 9 . 4 0}$ & $\mathbf{- 4 3 . 1 9}$ & -34.03 \\
\hline 7 & 40 & -76.13 & -23.29 & -61.33 & $\mathbf{- 5 6 . 5 5}$ & $\mathbf{- 4 6 . 9 7}$ & $\mathbf{- 3 9 . 3 3}$ & -34.94 \\
\hline 8 & 77 & -73.76 & -19.83 & -66.54 & $\mathbf{- 5 6 . 2 7}$ & $\mathbf{- 4 9 . 1 1}$ & $\mathbf{- 4 0 . 5 3}$ & -35.14 \\
\hline 9 & 72 & -73.22 & -31.41 & -65.17 & $\mathbf{- 5 8 . 8 8}$ & $\mathbf{- 5 2 . 1 2}$ & $\mathbf{- 4 6 . 6 9}$ & -42.59 \\
\hline 10 & 49 & -78.95 & -25.50 & -69.84 & $\mathbf{- 6 1 . 6 6}$ & $\mathbf{- 5 3 . 6 7}$ & $\mathbf{- 4 5 . 8 3}$ & -36.81 \\
\hline 11 & 40 & -71.60 & -34.04 & -64.78 & $\mathbf{- 6 2 . 1 9}$ & $\mathbf{- 5 7 . 3 4}$ & $\mathbf{- 4 6 . 9 7}$ & -41.85 \\
\hline $12-13$ & 15 & -64.78 & -36.92 & -64.38 & $\mathbf{- 6 2 . 7 8}$ & $\mathbf{- 5 6 . 8 1}$ & $\mathbf{- 4 6 . 9 7}$ & -39.29 \\
\hline Total & 363 & -78.95 & -19.83 & -65.38 & $\mathbf{- 5 8 . 2 2}$ & $\mathbf{- 5 0 . 5 4}$ & $\mathbf{- 4 3 . 9 0}$ & -36.50 \\
\hline
\end{tabular}

Table 2. Reference aortic pulse wave velocity values (PWV, $\mathrm{m} / \mathrm{s})$ in correlation to age

\begin{tabular}{|l|c|c|c|c|c|c|c|c|}
\hline \multicolumn{10}{|c|}{ PWV, m/s } \\
\hline Age (years) & \multirow{2}{*}{ N } & Min & Max & \multicolumn{5}{|c|}{$\%$} \\
\cline { 5 - 9 } & & & & 10 & $\mathbf{2 5}$ & $\mathbf{5 0}$ & $\mathbf{7 5}$ & 90 \\
\hline $3-5$ & 35 & 3.37 & 7.40 & 4.50 & $\mathbf{5 . 0 7}$ & $\mathbf{5 . 6 9}$ & $\mathbf{6 . 0 4}$ & 6.64 \\
\hline 6 & 35 & 3.89 & 7.65 & 4.81 & $\mathbf{5 . 3 2}$ & $\mathbf{5 . 5 8}$ & $\mathbf{6 . 3 7}$ & 7.04 \\
\hline 7 & 40 & 4.77 & 6.46 & 4.85 & $\mathbf{5 . 1 3}$ & $\mathbf{5 . 5 2}$ & $\mathbf{5 . 9 1}$ & 6.19 \\
\hline 8 & 77 & 4.09 & 7.65 & 4.97 & $\mathbf{5 . 3 8}$ & $\mathbf{5 . 7 3}$ & $\mathbf{6 . 4 0}$ & 6.87 \\
\hline 9 & 72 & 4.75 & 7.26 & 5.03 & $\mathbf{5 . 5 0}$ & $\mathbf{5 . 8 6}$ & $\mathbf{6 . 4 5}$ & 6.81 \\
\hline 10 & 49 & 4.78 & 7.85 & 5.06 & $\mathbf{5 . 5 7}$ & $\mathbf{6 . 1 6}$ & $\mathbf{6 . 7 1}$ & 7.20 \\
\hline 11 & 40 & 4.67 & 7.43 & 5.18 & $\mathbf{5 . 5 0}$ & $\mathbf{5 . 9 1}$ & $\mathbf{6 . 4 8}$ & 6.95 \\
\hline $12-13$ & 15 & 4.85 & 7.07 & 5.05 & $\mathbf{5 . 6 4}$ & $\mathbf{5 . 8 0}$ & $\mathbf{6 . 1 2}$ & 6.56 \\
\hline
\end{tabular}




\begin{tabular}{|l|l|l|l|l|l|l|l|l|}
\hline Total & 363 & 3.37 & 7.85 & 4.95 & $\mathbf{5 . 3 8}$ & $\mathbf{5 . 8 1}$ & $\mathbf{6 . 3 7}$ & 6.81 \\
\hline
\end{tabular}

Pic. 1. Dependence of PWV value $(\mathrm{m} / \mathrm{s})$ on the constitutive-exogenous obesity degree

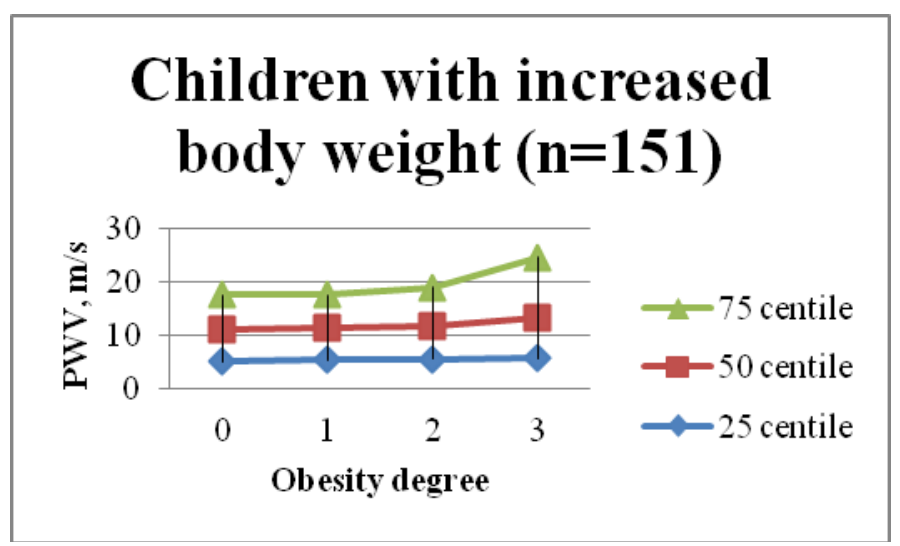

